\section{Chemoimmunotherapy with rituximab, cyclophosphamide and prednisolone in IgM paraproteinemic neuropathy: evidence of sustained improvement in electrophysiological, serological and functional outcomes}

There is ongoing debate as to the optimum treatment for IgM paraproteinemic neuropathy (PPN), with no treatment yet shown objectively to alter the long-term natural history of slow neurological decline. We present a case series of 25 patients with IgM PPN treated between August 2010 and October 2016 with standard R-CP (rituximab, cyclophosphamide, prednisolone) chemoimmunotherapy. This is the first report of detailed 2 -year serological, neurological and neurophysiological outcome data in patients treated prospectively with this regimen. The treatment was well tolerated and produced significant improvement for at least 2 years in several neurological, electrophysiological and serological outcomes.

Peripheral neuropathy is a well-recognized complication of IgM paraproteinemia, and occurs both in those with a monoclonal gammopathy of undetermined significance (MGUS) and in patients with an underlying lymphoproliferative disorder (LPD). Recent guidelines suggest that patients with renal or neurological complica- tions of paraproteinemia should be reclassified as having MGCS (monoclonal gammopathy of clinical significance $)^{1}$ to reflect the fact that they may merit treatment. In $50-60 \%$ of cases, the $M$ protein shows reactivity to a neural antigen known as myelin associated glycoprotein (MAG). ${ }^{2}$ There is accumulating evidence that the antiCD20 monoclonal antibody, rituximab, benefits a proportion of patients with IgM paraproteinemic neuropathy. ${ }^{3-6}$ However, there is insufficient evidence to assess the efficacy of rituximab alone compared with its use as part of a combined chemoimmunotherapy (CIT) regimen. International guidelines recommend that in patients with progressive disability due to anti-MAG neuropathy, immunosuppressive treatment should be considered as an alternative to rituximab monotherapy. ${ }^{7}$ A retrospective analysis of 45 PPN patients treated with a variety of treatment protocols also suggests a role for CIT. ${ }^{8}$ Finally, a recent demonstration of a high prevalence of mutated myeloid differentiation factor 88 (MYD88), which is strongly associated with Waldenstrom's macroglobulinemia (WM), in patients with anti-MAG neuropathy supports the use of treatment regimens effective in WM. ${ }^{9}$

Here we report a large prospective case series of patients with progressive disability due to confirmed IgM paraproteinamic neuropathy treated with standard R-CP chemoimmunotherapy. R-CP is standard R-CVP, minus

Table 1. Patients' characteristics $(n=25)$.

Mean age at presentation, years (range)

$68(50-81)$

Gender, n. (\%)

Male: 20 (80)

Female: 5 (20)

Duration of neuropathy symptoms prior to treatment

Median 5 years (range 1-20)

Hematologic diagnosis, n. (\%)

IgM MGCS: 16 (64)

WM: $8(32)$

CLL : 1 (4)

Paraprotein concentration $(\mathrm{g} / \mathrm{L})$

Median 4.7 (range 1.6-17.9)

Anti-MAG antibody status

Anti-MAG positive: 18 (72) (>1,000 Bühlmann titer units)

Anti-MAG negative: 7 (28)

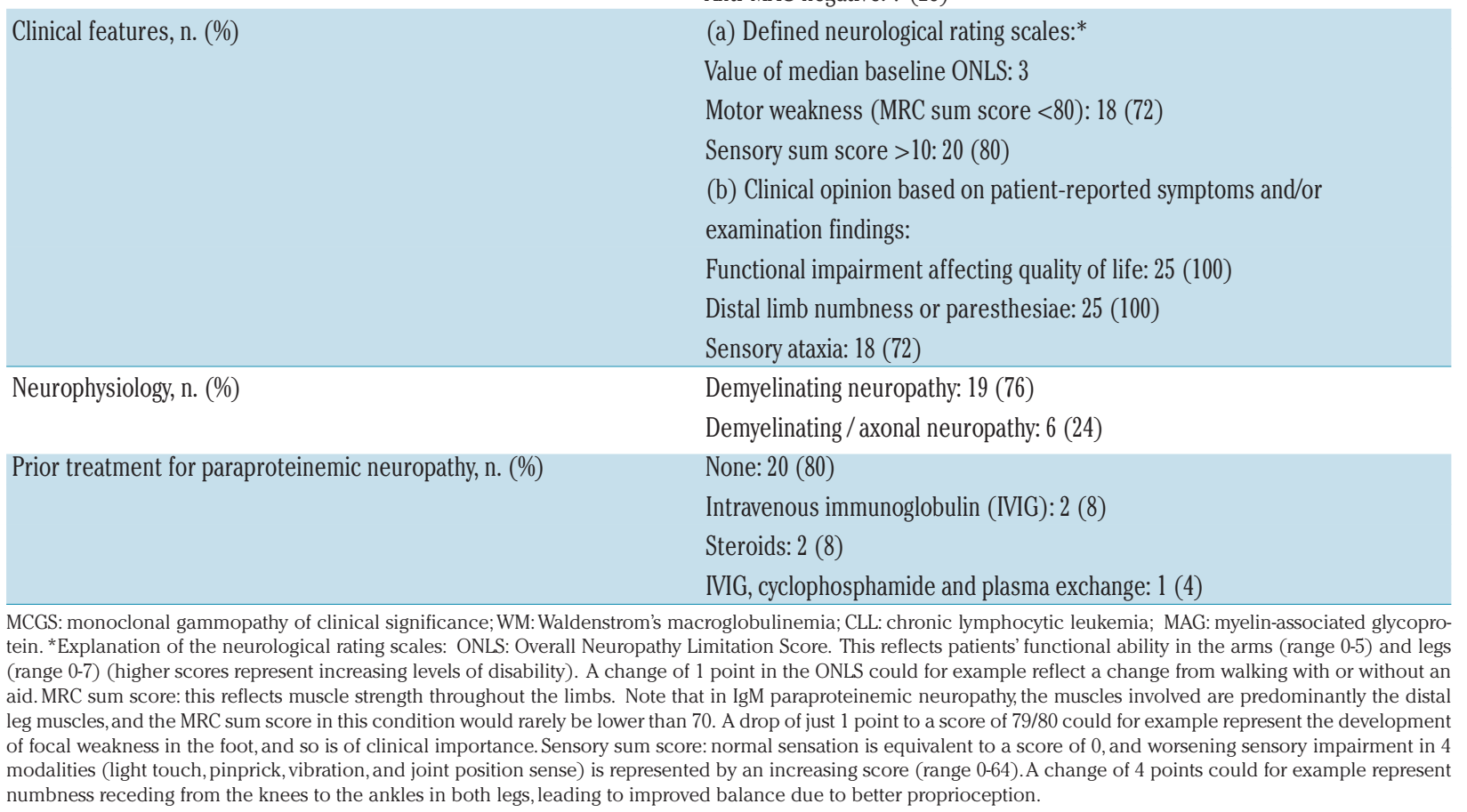


vincristine to avoid neurological toxicity. It is very similar to the DRC (dexamethasone, rituximab, cyclophosphamide) protocol ${ }^{10}$ used frequently in the treatment of WM, and similar to immunosuppressive protocols used in rheumatological disorders. All patients had IgM paraproteinemia, fulfilling criteria either for WM or for MGCS, together with electrophysiological evidence of neuropathy. Comprehensive assessment was undertaken to establish a likely causal relationship between the IgM paraprotein and the neuropathy, and to exclude other conditions such as diabetic or vasculitic neuropathy.

In total, 33 patients have started treatment. We report results from 25 patients at one year and from 18 patients at 2 years (i.e., those who have reached these time points) (Table 1).

Baseline investigations included bone marrow biopsy and computed tomography (CT) body scan, as well as full standard hematology and biochemistry screen, serum protein electrophoresis, and anti-MAG antibody titers using an enzyme-linked immunosorbent assay (ELISA). MYD88 testing was not carried out as the patients presented here pre-dated the routine clinical use of this assay. Following fully informed written consent, treatment comprised six cycles (every 21 days) of the following regimen: rituximab $375 \mathrm{mg} / \mathrm{m}^{2}$ intravenously and cyclophosphamide $750 \mathrm{mg} / \mathrm{m}^{2}$ intravenously on day 1 ; and prednisolone $50 \mathrm{mg} / \mathrm{m}^{2}$ orally on days 1-5. All patients completed the full treatment protocol and all received prophylaxis for at least 6 months post treatment to reduce treatment-related complications.

The treatment protocol was generally well tolerated.
Complications were rare: one flu pneumonitis, one dental abscess, and seven other minor complications, all of which resolved fully. This tolerance compares favorably with the larger rituximab monotherapy randomized controlled trial in which eight serious adverse events occurred in 26 patients. ${ }^{4}$

It has recently emerged that, out of 33 patients who have been treated in total, three patients have since developed IDH1 (isocitrate dehydrogenase) negative glioblastoma. Two patients were anti-MAG positive with MGCS, one patient was anti-MAG negative with underlying WM. Given that the incidence of glioblastoma multiforme (GBM) is 3 in 100,000 , this is concerning. At this stage it is unclear whether the GBM cases: (i) are a random occurrence; (ii) relate to the known association between WM and GBM; ${ }^{11}$ (iii) reflect an unknown shared risk factor between IgM PPN and GBM; or (iv) relate to the use of CIT in IgM PPN. The last point seems unlikely as, firstly, epidemiological studies of GBM show no link between immunosuppression and GBM incidence, ${ }^{12}$ and, secondly, this CIT regimen has been used widely in hematology and rheumatology for over 20 years, with no suggestion of a link with GBM.

The serology results in individual patients are shown in Figure 1 . At 2 years post treatment completion, paraprotein concentration fell compared to baseline in 17 of 18 (94\%) patients (Figure 1A). Median paraprotein concentration fell from $4.7 \mathrm{~g} / \mathrm{L}$ at baseline to $2.0 \mathrm{~g} / \mathrm{L}$ at 2 years $\left(P=0.000^{*}\right)$. Twelve of 13 anti-MAG positive patients showed a reduction in anti-MAG titer compared to baseline (Figure 1B). Median anti-MAG titer at baseline was

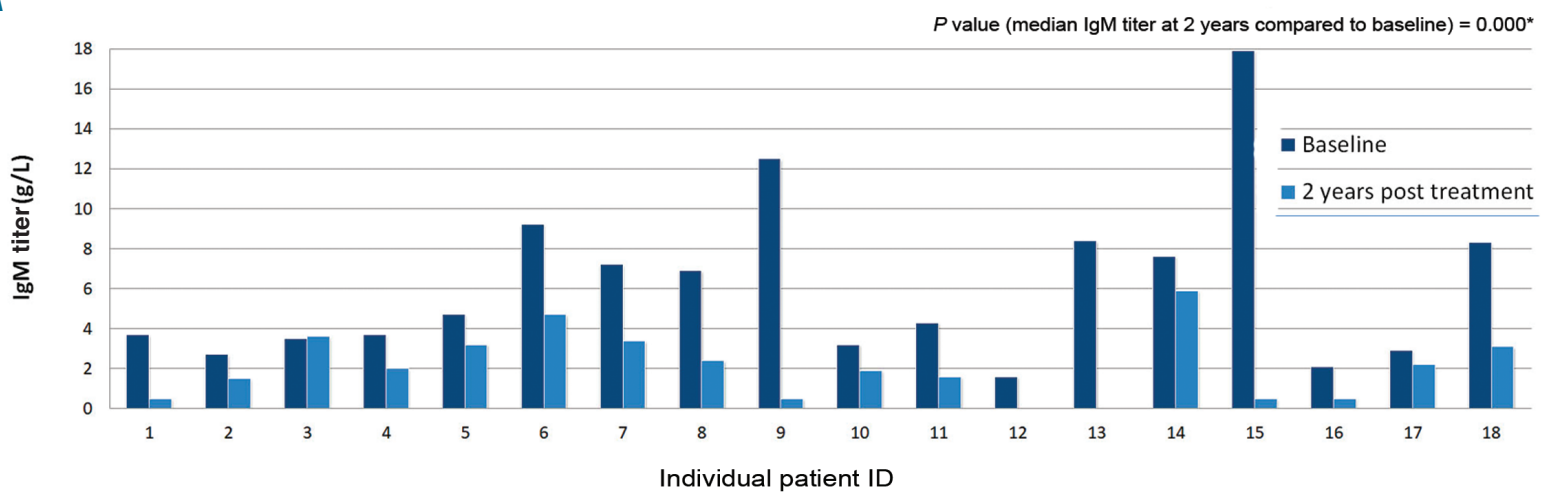

B

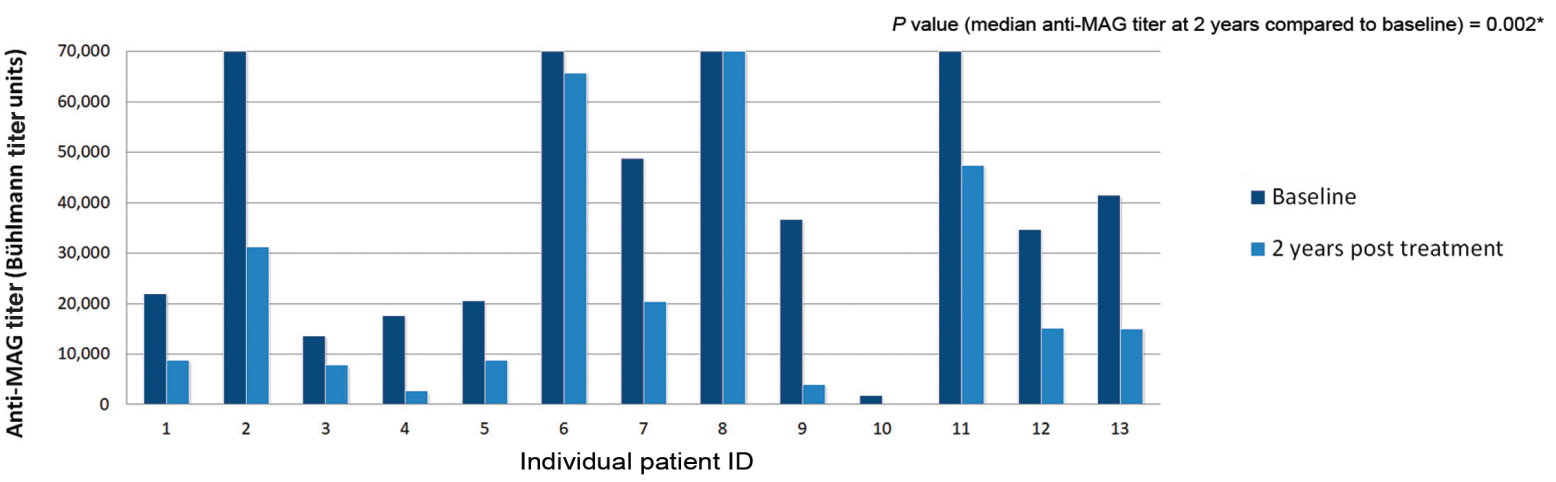

Figure 1. Response of IgM paraprotein and anti-myelin associated glycoprotein (MAG) titers to R-CP (rituximab, cyclophosphamide, prednisolone) chemoimmunotherapy treatment. (A) IgM titer at baseline and 2 years post treatment. (B) Anti-MAG titer at baseline and 2 years post treatment. Patients 1-13 in Figure $1 \mathrm{~A}$ are the anti-MAG positive patients, and these correspond to patients 1-13 in Figure 1B 
38,956 Bühlmann titer units (BTU), falling to $14,783 \mathrm{BTU}$ at 2 years $(P=0.002 *)$.

Neurological symptoms and disability were measured using the Overall Neuropathy Limitation Score (ONLS). ${ }^{13}$ A 1 point change in ONLS reflects a meaningful change in patient function, e.g., the difference between walking with or without an aid. Median ONLS showed a significant improvement from 3 at baseline to 2 at 1 year $\left(P=0.006^{*}\right)$ and to 1 at 2 years $(P=0.053)$. Motor function was quantified using the Medical Research Council (MRC) Sum Score of muscle power (range $0-80),{ }^{14}$ in which, e.g., a loss of just 1 point equates to the development of a partial footdrop. Eighteen patients (72\%) had motor weakness at baseline, and 13 have 2-year followup data: MRC sum score in these patients showed a significant improvement in the median score (79) compared to baseline (76) $\left(P=0.031^{*}\right)$, and 9 of 13 patients $(69 \%)$ improved by at least 1 point. Sensory Sum Score reflects the extent of sensory loss in limbs: ${ }^{14}$ e.g., a change of 4 points reflects numbness receding from the knees to the ankles in both legs. Sensory Sum Score improved by at least 4 points in 9 of $18(50 \%)$ patients compared to baseline. On direct questioning about their symptoms 2 years post treatment, $65 \%$ of patients reported either improvement (8 of 17 patients, $47 \%$ ) or stabilization (3 of 17 patients, $18 \%$ ).

Electrophysiology provides an objective, quantifiable measure of nerve function, which allows us to demon-

A

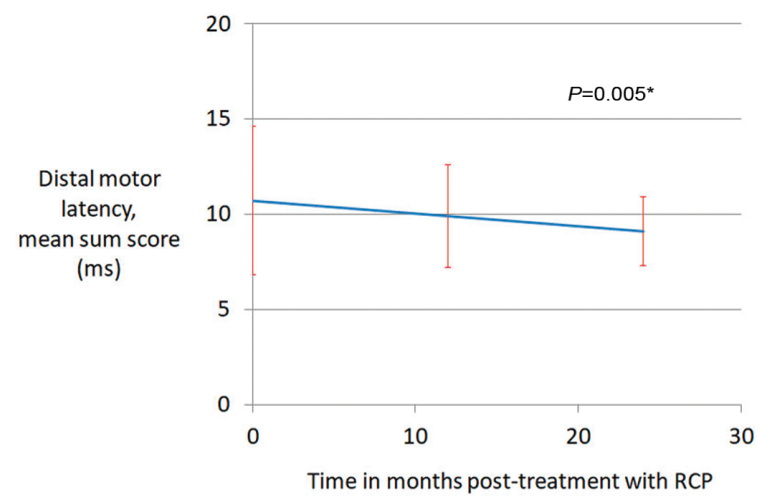

C

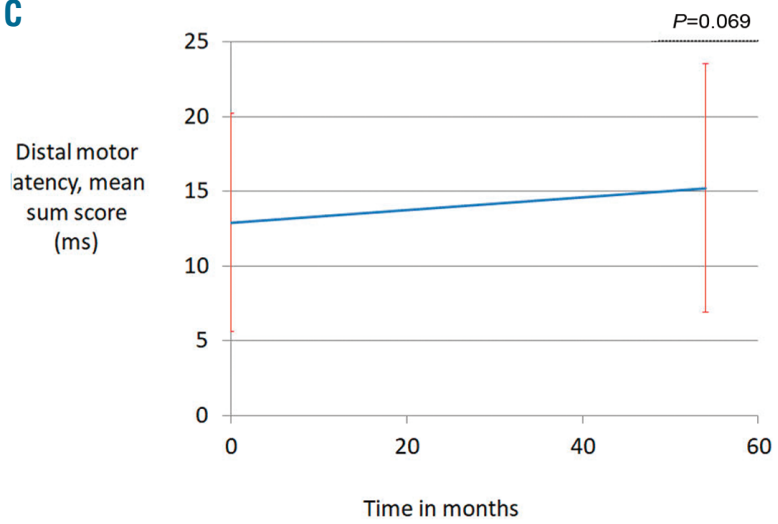

strate whether the neuropathy is progressing, stable or improving. Our electrophysiological studies (see Figure 2 for definitions) showed that the mean distal motor latency (DML) sum score improved from 10.7 milliseconds (ms) at baseline to $9.1 \mathrm{~ms}$ at 2 years $\left(P=0.005^{*}\right)$ (Figure 2A). Sensory nerve action potential (SNAP) sum score also showed a trend to improvement, increasing from 11.7 microvolts (uv) to 15.7 uv at 2 years, $(P=0.26)$ (Figure 2B). Additional neurophysiology is presented in eight off-protocol patients, as there is little in the literature regarding long-term changes in neurophysiology in patients with IgM PPN. In these patients, both motor and sensory nerve conduction studies deteriorated over a 54month period. The mean SNAP sum score significantly worsened from a baseline of 17.4 uv to 8.2 uv at 54 months $\left(P=0.028^{*}\right)$ (Figure 2D). The mean DML sum score also deteriorated from a baseline mean of $12.9 \mathrm{~ms}$ to $15.1 \mathrm{~ms}$ over the same period $(P=0.069)$ (Figure $2 \mathrm{C}$ ). The clinical importance of these findings is notable: we demonstrate that untreated patients show a progressive deterioration in neurophysiology which, despite not being directly comparable due to the differing follow-up times, is in contrast to those treated with this CIT regimen, who showed a significant improvement in motor response and a trend towards sensory improvement 2 years after treatment.

Our case series is not sufficiently large to study the factors which may predict individual patient responses by

B

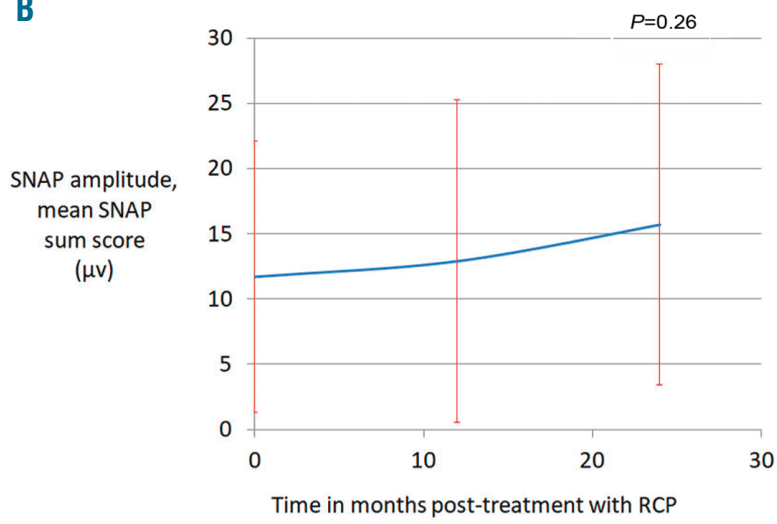

D

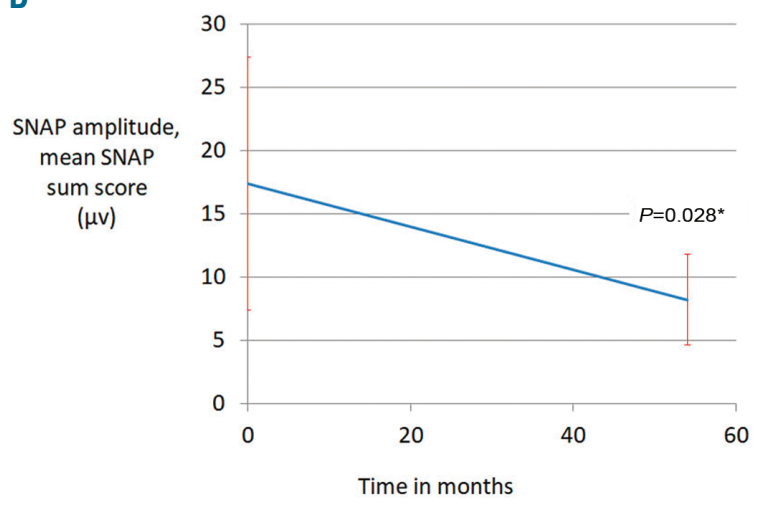

Figure 2. Motor and sensory electrophysiological changes 2 years post R-CP (rituximab, cyclophosphamide, prednisolone) chemoimmunotherapy treatment compared with historical off-treatment group. (A) Distal motor latency: mean sum scores at baseline, 12 and 24 months in protocol group: $n=23$ at 12 months and $n=15$ at 24 months. (B) Sensory nerve action potentials: mean SNAP sum score at baseline, 12 and 24 months in protocol group: $n=23$ at 12 months and $n=15$ at 24 months. (C) Distal motor latency: mean sum score trend in historical off-protocol group over a 54-month period ( $n=8$ ). (D) Sensory nerve action potentials: mean SNAP sum score trend in historical off-protocol group over a 54-month period $(n=8)$. Error bars represent the standard deviation. DML mean sum score: mean of right median and ulnar nerve distal motor latencies; Mean SNAP sum score: mean of right median, ulnar and radial nerve sensory nerve action potentials; DML: distal motor latency; SNAP: sensory nerve action potential; ms: milliseconds; $\mu \mathrm{v}$ : microvolts. 
sub-group analysis. We plan to undertake a further analysis at a later time point with a larger cohort of patients in order to correlate neurological response with individual patient factors.

This is the first report in IgM paraproteinemic neuropathy of the prospective use of a standardized CIT protocol with detailed outcome measures at 2 years post treatment. Although not a formal randomized comparison, our outcome data compare favorably with the rituximab monotherapy data from the literature. Based on more than 200 rituximab-treated patients, the average number of patients responding to treatment is $30-50 \%$ at time points of up to one year, ${ }^{6}$ whereas our data illustrate improvements in 10 of $18(55 \%)$ patients for ONLS, 9 of 13 patients $(69 \%)$ for MRC sum score, 9 of 18 patients $(50 \%)$ for sensory sum score and 11 of 17 patients $(65 \%)$ for PROMS, at a longer time point of 2 years. However, despite the fact that CIT has been used safely in hematooncology and rheumatology for decades, this case series raises the possibility of an increased incidence of malignant primary central nervous system tumor in patients with IgM PPN treated with such protocols. It is important that other centers treating IgM PPN patients with CIT be vigilant for the occurrence of malignancy, particularly GBM. Further epidemiological study is needed to look at the incidence of GBM in other case series of IgM PPN, with or without treatment, and in patients treated with CIT for other indications.

Nancy T. H. Colchester, ${ }^{1}$ David Allen, ${ }^{2}$ Haider A. Katifi, ${ }^{1}$ Tracy Burt, ${ }^{3}$ Robert N. Lown, Ashwin A. Pinto and Andrew S. Duncombe

'Department of Neurology, Wessex Neurological Centre, University Hospital Southampton; ${ }^{2}$ Department of Neurophysiology, Wessex Neurological Center, University Hospital Southampton and

${ }^{3}$ Department of Haematology, University Hospital Southampton, Southampton, UK

Correspondence:

NANCY T. H.COLCHESTER - nancy.colchester@uhs.nhs.uk

doi:10.3324/haematol.2019.243139

Disclosures: no conflicts of interests to disclose.

Contributions: NC analyzed the results, wrote sections of the manuscript and conducted neurological assessments; DA performed, analyzed and wrote up all the neurophysiology; HK oversaw patient selection and reviewed from a neurology perspective; TB designed the database and collated the serological results; RL helped supervise treatment. AP contributed patients for assessment; $A D$ wrote the treatment protocol and guideline, assessed patient suitability, supervised treatment and wrote sections of the manuscript. All authors contributed to reviewing and editing the manuscript.
Acknowledgments: the authors would like to thank the patients for their engagement in the treatment and outcome monitoring. We also thank Rutaba Eshita, Mazen Sabah, Chinar Osman and Annamaria Kiss-Csenki, Consultant Neurologists at University Hospital Southampton (RE and CO) and at Hampshire Hospitals NHS Foundation Trust (MS and AK) for their work in performing the neurological assessments. We also thank Ian Galea, Associate Professor in Neurology at the University of Southampton and Consultant Neurologist, for his advice regarding the statistical analysis.

\section{References}

1. Fermand JP, Bridoux F, Dispenzieri A, et al. Monoclonal gammopathy of clinical significance: a novel concept with therapeutic implications. Blood. 2018;132(14):1478-1485.

2. Nobile-Orazio E, Manfredini E, Carpo M, et al. Frequency and clinical correlates of anti-neural IgM antibodies in neuropathy associated with IgM monoclonal gammopathy. Ann Neurol. 1994;36(3):416424.

3. Dalakas MC, Rakocevic G, Salajegheh M, et al. Placebo-controlled trial of rituximab in IgM anti-myelin-associated glycoprotein antibody demyelinating neuropathy. Ann Neurol. 2009;65(3):286-293.

4. Leger JM, Viala K, Nicolas G, et al. Placebo-controlled trial of rituximab in IgM anti-myelin-associated glycoprotein neuropathy. Neurology. 2013;80(24):2217-2225

5. Lunn MP, Nobile-Orazio E. Immunotherapy for IgM anti-myelin associated glycoprotein paraprotein-associated peripheral neuropathies. Cochrane Database Syst Rev. 2016;10:CD002827.

6. Dalakas MC. Rituximab in anti-MAG neuropathy: more evidence for efficacy and more predictive factors. J Neurol Sci. 2017;377:224226.

7. D'Sa S, Kersten MJ, Castillo JJ, et al. Investigation and management of IgM and Waldenstrom-associated peripheral neuropathies: recommendations from the IWWM-8 consensus panel. Br J Haematol. 2017;176(5):728-742

8. Hospital M, Viala K, Dragomir S, et al. Immunotherapy-based regimen in anti-MAG neuropathy: results in 45 patients. Haematologica. 2013;98(12):e155-157.

9. Vos JM, Notermans NC, D'Sa A, et al. High prevalence of the MYD88 L265P mutation in IgM anti-MAG paraprotein-associated peripheral neuropathy. J Neurol Neurosurg Psychiatry. 2018; 89(9):1007-1009.

10. Dimopoulos MA, Agagnostopulos A, Kyrtsonis MC, et al. Primary treatment of Waldenstrom's macroglobulinaemia with dexamethasone, rituximab and cyclophosphamide. J Clin Oncol. 2007; 25(22):3344-3349.

11. Lamaida E, Caputi F, Rapana A, Bracale C, Graziussi G. Waldenstrom's macroglobulinaemia associated with glioblastoma. A case report. Rev Neurol (Paris). 1996;152(10):637-639.

12. Thakkar JP, Dolecek TA, Horbinski C, et al. Epidemiologic and molecular prognostic review of glioblastoma. Cancer Epidemiol Biomarkers Prev. 2014;23(10):1985-1996.

13. Van Nes SI, Faber CG, Merkies IS. Outcome measures in immunemediated neuropathies: the need to standardise their use and to understand the clinimetric essentials. J Peripher Nerv Syst. 2008; 13(2):136-147

14. RMC Trial Group. Randomised controlled trial of methotrexate for chronic inflammatory demyelinating polyradiculoneuropathy (RMC trial): a pilot, multicentre study. Lancet Neurol. 2009;8(2):158-164. 